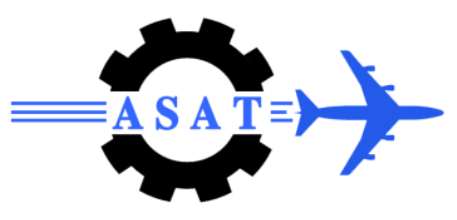

\title{
Vehicle Tracking System Using GPS/INS Integrated with Kalman Filter
}

\author{
Marwa Algamal ${ }^{*}$, Gamal Selim ${ }^{\dagger}$, and Shawki Eid ${ }^{\dagger}$
}

\begin{abstract}
Efficient vehicle tracking is critical in busy metropolitan areas. A new innovative tracking technique can improve the system performance for the cab companies which are used to serve inhabitants. In fact, detecting the exact position of the vehicle can help to direct the user to the nearest vehicle for the intended customer. It also provides a fast service which helps to reduce the traffic loads. In this paper, a combination between global positioning system (GPS) and Inertial navigation system (INS) integrated with Kalman filter will be developed in order to enhance the vehicle tracking performance. The system can be applied in many applications such as water vehicles, aircrafts, ships and cars. In this work, the target of this application is Taxi Companies vehicular tracking.
\end{abstract}

\section{Introduction}

There is an extensive literature on estimating the position of vehicles using Kalman Filter integrated with global positioning system and inertial navigation system [1]-[2]. Errors like (tropospheric delays like ionospheric delays and atmospheric delays, Multipath effects etc.) affecting the GPS signals as they travel from satellite to the user on Earth. These errors degrade the accuracy of GPS position. INS errors are attributed due to the existence errors in inertial sensors (instrument errors). Therefore, a linear recursive filtering technique called Kalman filter is used for greater accuracy in estimating the position of the vehicle. This can be done by the proposed algorithm. It is verified by using MATLAB computer simulation and the results obtained from proposed Kalman filter technique give a good accuracy in vehicle position [3].

\section{System Description}

\section{City Cab Application}

To provide good customer service in the taxi business, fast and efficient dispatching is a critical factor. Taxi dispatching systems have relied traditionally on good staff and teamwork. A new application is using a GPS/INS integrated with Kalman filter. Through the use of this computerized satellite-based system, costs effective and efficient servicing system are achieved [4]. Figure 1 describe the system proceeding.

\footnotetext{
Department of Computer Engineering, Arab Academy for Science Technology and Maritime Transport, Mero.m.gamal@ hotmail.com

${ }^{\dagger}$ Prof., Department of Computer Engineering, Arab Academy for Science Technology and Maritime Transport
} 


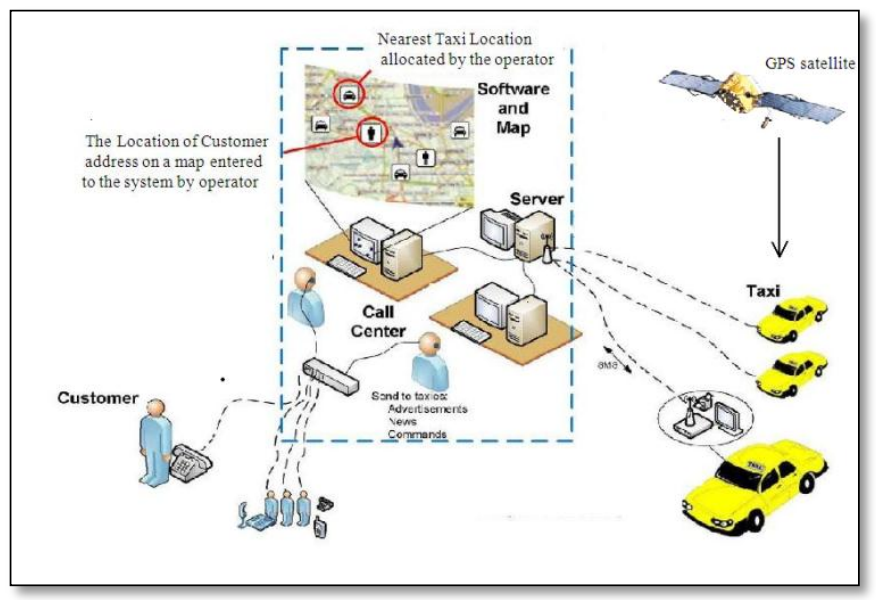

Figure 1: Navigation Systems for Taxies

\section{System Proceeding}

1. Passenger call for a cab, a customer service officer take address and the pick-up time needed.

2. The operator stores the customer location on the map.

The location and status of all vehicles are appeared in the center screen on a street level map. It uses the GPS/INS capability to show the accurate position at real-time of their fleet. Vehicle positions on the map have different colors according to whether they are Clear, Assigned, Loaded, Busy, Soon-to-Clear or Off Service.

1. Nearest Taxi location to the passenger pick-up point is allocated by the operator.

2. The booking details are sent to the assigned driver. Then, it will appear on a display terminal LCD in the driver's car [4].

\section{A. Global Positioning Systems (GPS)}

GPS was developed by the US Department of Defense to provide all-weather round-the-clock navigation capabilities for military ground, sea, and air forces. Since its implementation, GPS has also become an integral asset in numerous civilian applications and industries around the globe, including recreational uses (e.g. boating, aircraft, hiking), corporate vehicle fleet tracking, and surveying. GPS employs 24 spacecraft in $20,200 \mathrm{~km}$ circular orbits. The satellites provide two different signals that provide different accuracies. Coarse-Acquisition (C/A) code is intended for civilian use, and is deliberately degraded. The accuracy using a typical civilian GPS receiver with C/A code is typically about 100 meters. The military's Precision $(\mathrm{P})$ code is not corrupted, and provides positional accuracy to within approximately 20 meters.

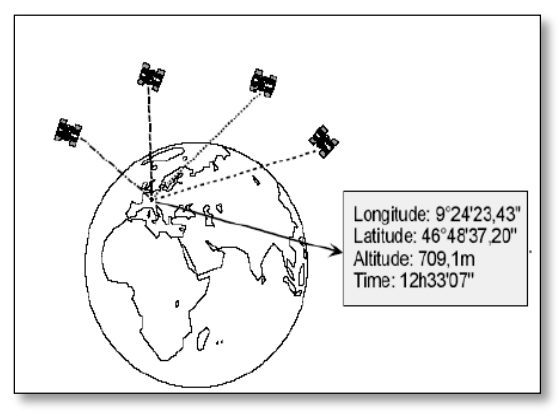

Figure 2: The basic Function of GPS 
The total GPS configuration is comprised of three distinct segments:

- The Space Segment: satellite orbited by earth.

- The Control Segment: stations positioned on the earth's equator to control the satellite.

- The User Segment: Anybody that receives and uses the GPS signal.

\section{GPS Errors Sources}

There are several sources of error that degrade the GPS position from few meters to hundreds of meters. These errors are:

- Noise errors.

- Ephemeris errors occur when the GPS message does not transmit the correct satellite location.

- Satellite clock errors affect both C/A and P code.

- Ionospheric and tropospheric delays are introduced due to the atmosphere like the changes in temperature, pressure and humidity associated with weather changes and this leads to a phase lag in calculation of the pseudo range.

- Control segment mistakes due to computer or human error.

- Receiver errors from software or hardware failures can cause blunder errors of any size [5].

\section{B. Inertial Navigation Systems (INS)}

An Inertial Navigation System (INS) is a navigation aid that uses a computer, motion sensors (accelerometers) and rotation sensors (gyroscopes) to continuously calculate the position, orientation, and velocity of a moving object without the need for external references. It is used on vehicles such as cars, ships, aircrafts, submarines, guided missiles, and spacecraft [6].

\section{Errors in the INS}

Most INS errors are attributed to the inertial sensors (instrument errors). These are the residual errors exhibited by the installed gyros and accelerometers following calibration of the INS gyroscopes, which are used as attitude sensors in inertial navigation, also called as inertial grade. There are many types of gyroscope designs such as momentum wheels gyro, rotating multisensory gyroscopes, laser gyroscopes, etc. The common error sources for gyroscopes are: output bias, input axis misalignment and input/output non-linearity.

Acceleration sensors also have several designs such as, pendulous accelerometers, strainsensing accelerometers, Micro-machined silicon accelerometers MEMS etc. The main error sources for accelerometers are:

- Bias of an accelerometer: it is the offset of its output signal from the true value, in $m / s^{2}$. A constant bias error of $\varepsilon$, when double integrated, causes an error in position which grows with time.

- Parameter instabilities (i.e., turn-on and drift) [7].

\section{The Kalman Filter (KF)}

The Kalman filter is a multiple-input, multiple-output digital filter that can optimally estimate, in real time, the states of a system based on its noisy outputs. These states are all the variables needed to completely describe the system behavior as a function of time (such as position, velocity, voltage levels, and so forth). In fact, one can think of the multiple noisy outputs as a multidimensional signal plus noise, with the system states being the desired unknown signals. The Kalman filter then filters the noisy measurements to estimate the desired signals. The estimates are statistically optimal in the sense that they minimize the mean-square estimation error [8]. 


\section{GPS/INS Integrated with Kalman Filter Algorithm}

The Kalman filter is essentially a set of mathematical equations that implement a predictorcorrector type estimator that is optimal in the sense that it minimizes the estimated error covariance when some presumed conditions are met. Since the time of its introduction, the Kalman filter has been the subject of extensive research and application, particularly in the area of autonomous or assisted navigation [5]. The Kalman filter addresses the general problem of trying to estimate the state $\mathrm{x} \in \mathrm{R}^{\mathrm{n}}$ of a discrete-time controlled process that is governed by the linear stochastic difference equation:

$$
x_{k}=A x_{k-1}+B u_{k-1}+w_{k-1}
$$

with a measurement $\mathrm{z} \in \mathrm{R}^{\mathrm{m}}$ that is

$$
z_{k}=H x_{k}+v_{k}
$$

where $z_{k}$ is the observable at time $k$, and $H$ is the measurement matrix. The random variables $\mathrm{w}_{\mathrm{k}}$ and $\mathrm{v}_{\mathrm{k}}$ represent the process and measurement noise respectively [9].

$$
\begin{aligned}
& p(w) \sim N(0, Q) \\
& p(v) \sim N(0, R)
\end{aligned}
$$

In practice, the process noise covariance $\mathrm{Q}$ and measurement noise covariance $\mathrm{R}$ matrices will change with each time step or measurement. However they are constant like in (5)[10].

$$
\begin{gathered}
Q=\left[\begin{array}{ll}
1 & 0 \\
0 & 1
\end{array}\right] \\
R=1
\end{gathered}
$$

The $\mathrm{n} \times \mathrm{n}$ matrix $\mathrm{A}$ in (7) is the system matrix in the difference equation (1). It relates the state at the previous time step K-1 to the state at the current step $\mathrm{K}$, in the absence of either a driving function or process noise. In practice A will change in each time step [8].

$$
A=\left[\begin{array}{ll}
0 & 1 \\
0 & 0
\end{array}\right]
$$

The $n \times 1$ matrix $\mathrm{B}$ in (8) relates the control input $\mathrm{u} \in R$ to the state $\mathrm{x}$ :

$$
B=\left[\begin{array}{l}
0 \\
0
\end{array}\right]
$$

The $m \times n$ matrix $\mathrm{H}$ in (9) in the measurement equation (2) relates the state to the measurement $Z_{k}$. In practice $\mathrm{H}$ will be constant.

$$
H=\left[\begin{array}{ll}
1 & 1
\end{array}\right]
$$

The Kalman filter estimates a process by using a form of feedback control: the filter estimates the process state at some time and then obtains feedback in the form of (noisy) measurements. As such, the equations for the Kalman filter fall into two groups: time update equations and 
measurement update equations as shown in figure 1 Discrete Kalman filter time update equations (10) and (11) are given as

$$
\begin{gathered}
\widehat{x}_{k}^{-}=A \widehat{x}_{k-1}+B u_{k-1} \\
P_{k}^{-}=A P_{k-1} A^{T}+Q
\end{gathered}
$$

Time update equations project the state and covariance estimates forward from time step k-1 to step k. A and B are from equation (1), while Q is from is from equation (3). Initial conditions for the filter are discussed in the earlier references. Discrete Kalman filter measurement update equations (12) (13) and (14) are given below:

$$
\begin{gathered}
K_{k}=P_{k}^{-} H^{T}\left(H P_{k}^{-} H^{T}+R\right)^{-1} \\
\widehat{x}_{k}=\widehat{x}_{k}^{-}+K_{k}\left(z_{k}-H \widehat{x}_{k}^{-}\right) \\
P_{k}=\left(I-K_{k} H\right) P_{k}^{-}
\end{gathered}
$$

The time update equations are responsible for projecting forward (in time) the current state and error covariance estimates to obtain the a priori estimates for the next time step. The measurement update equations are responsible for the feedback [3][11]. Indeed the final estimation algorithm resembles that of a predictor-corrector algorithm for solving numerical problems. The first task during the measurement update is to compute the Kalman gain $K_{k}$. The next step is to actually measure the process to obtain $Z_{k}$, and then to generate an $a$ posteriori state estimate by incorporating the measurement as in equation (13). The final step is to obtain an a posteriori error covariance estimate via equation (14). After each time and measurement update pair, the process is repeated with the previous a posteriori estimates used to project or predict the new a priori estimates [3].

\section{Filter Parameters}

In the actual implementation of the filter, the measurement noise covariance $\mathrm{R}$ is usually measured prior to operation of the filter. Measuring the measurement error covariance $\mathrm{R}$ is generally practical because we need to be able to measure the process while operating the filter so we should generally be able to take some off-line sample measurements in order to determine the covariance of the measurement noise The determination of the process noise covariance $\mathrm{Q}$ is generally more difficult as we typically do not have the ability to directly observe the process we are estimating. Sometimes a relatively simple. In closing we note that under conditions where $\mathrm{Q}$ and $\mathrm{R}$ are in fact constant, both the estimation error covariance PK and the Kalman gain KK will stabilize quickly and then remain constant [12].

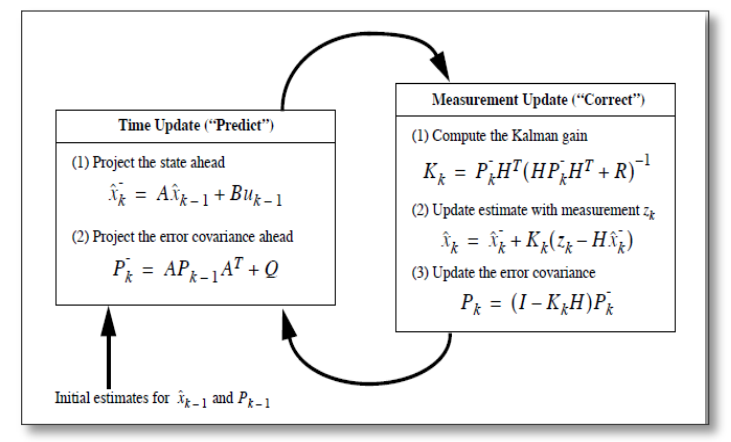

Figure 3: A complete picture of the operation of the Kalman filter 


\section{Simulation Results}

In this section we describe our simulation results. We can see that the Kalman filter provides a simple algorithm that can easily lend itself to integrated systems and requires only adequate statistical models of the state variables and associated noises for its optimal performance. Figure 4 shows the simulation from the noisy data of GPS and errors assumed to be from atmospheric changes. In figure 5 it appear that INS has also errors assumed to be happened from the sensors misalignment. The integration of the two systems through Kalman Filter in figure 6 corrects errors and gives an accurate prediction for the next position of the vehicle.

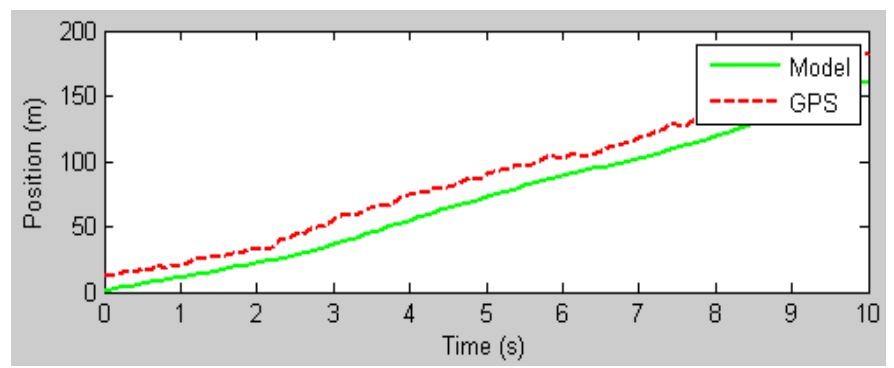

Figure 4: GPS estimation with Kalman Filter.

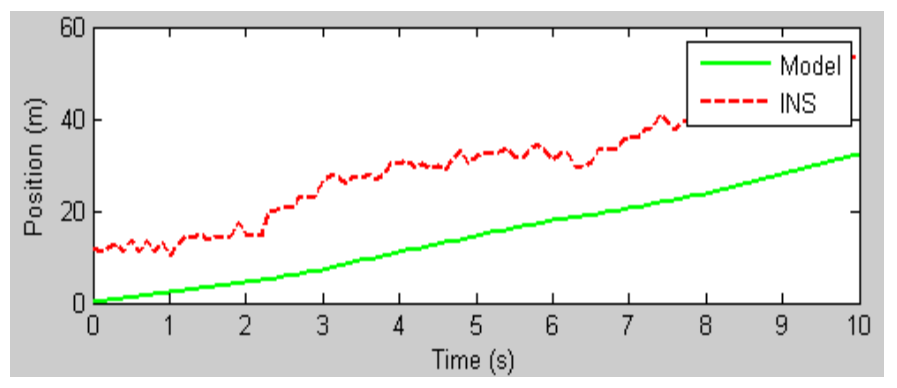

Figure 5: INS errors estimation with Kalman Filter.

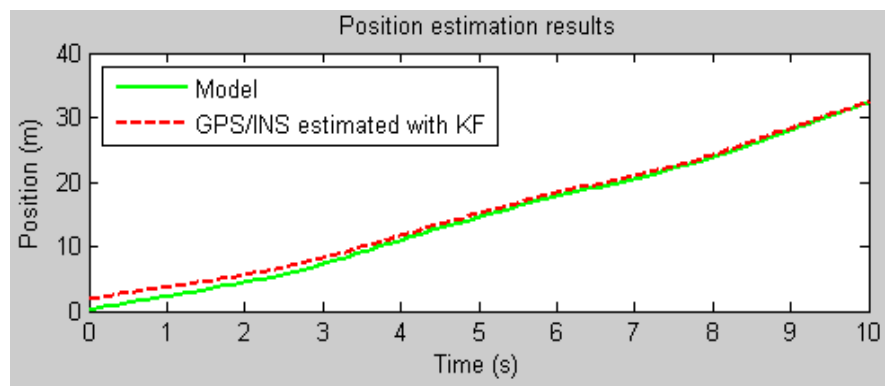

Figure 6: GPS/INS estimation with Kalman Filter.

Control theory in general and Kalman filtering in particular, provide a theoretical framework for combining information from various sensors. One of the most common alternative sensors is a satellite navigation radio, such as GPS. By properly combining the information from an INS and the GPS system (GPS/INS), the errors in position and velocity are stable. Furthermore, INS can be used as a short-term fallback while GPS signals are unavailable, for example when a vehicle passes through a tunnel [6]. 


\section{Conclusion}

A proposed approach has been developed for the received data from GPS/INS, is used to improve the tracking system. This method uses Kalman filter to decrease the error of the vehicle position. The total performance of the system improved the overall navigation performance and can give an accurate position to the tracked object. This study will be used to improve the system performance for the cab companies, by locating the exact position of the vehicle by GPS/INS integrated with Kalman filter and to eliminate that discussed before and give a good prediction to the next position of the vehicle using Kalman filter.

\section{References}

[1] W. Kao, "Integration of GPS and Dead-Reckoning Navigation Systems", In Proc. IEEE Vehicle Navigation and Information Systems Conference, SAE, pp.635-643, 1991.

[2] S. Panzieri, F. Pascucci, G. Ulivi, "An Outdoor Navigation System using GPS and Inertial Platform", IEEE/ASME Transactions on Mechatronics, Vol. 7,No.2, pp. 134-42, 2002.

[3] B.L. Malleswari, I.V. MuraliKrishna et al., "The Role of Kalman Filter in the Modeling of GPS Errors", Journal of Theoretical and Applied Information Technology, pp 95- 98, 2009.

[4] Yumoto, N., and Y. Liu. "GPS Technology in Advanced Traffic Information Systems." Proceedings of the International Conference on Advanced Technologies in Transportation and Traffic Management, Singapore: pp 391-398, 1994.

[5] Grewel.M. M S, Lawrence R. Weill, Angus P. Andrews, Global Positioning System, Inertial Navigation and Integration, $1^{\text {st }}$ edition (New York: John Wiley \& Sons), 2001.

[6] http://en.wikipedia.org/wiki/Inertial_navigation_system.

[7] O.J.Woodman ,"An introduction to inertial navigation", Technical reports published by the University of Cambridge,pp.14-15,2007.

[8] L. J. Levy, "The Kalman Filter: Navigation's Integration Workhorse", The Johns Hopkins University, Applied Physics Laboratory,2002.

[9] S.Haykin, "Kalman Filter and Neural Network", p 2-4 , 2001.

[10] Peter S Maybeck, Stochastic Models estimation and control, 1st Ed, (New York: PTH), 2001.

[11] G. Welch, G. Bishop “An Introduction to the Kalman Filter”, pp 19-24, 2001.

[12] Bozic. S, M, Digital and Kalman Filtering: An Introduction to Discrete-Time Filtering and Optimum Linear Estimation, $2^{\text {nd }}$ edition (New York: John Wiley\& Sons), 1999.

[13] D. Hohman, T. Murdock, et. al., "GPS Roadside Integrated Precision Positioning System", In Proc. IEEE Position Location and Navigation Symposium, pp. 221-230, 2000 . 